\title{
Nonequilibrium spin density and spin-orbit torque in a three-dimensional topological insulator/antiferromagnet heterostructure
}

\author{
S. Ghosh* and A. Manchon ${ }^{\dagger}$ \\ King Abdullah University of Science and Technology (KAUST), \\ Physical Science and Engineering Division (PSE), Thuwal 23955, Saudi Arabia
}

(Received 7 March 2019; published 10 July 2019)

\begin{abstract}
We study the behavior of nonequilibrium spin density and spin-orbit torque in a topological insulator/antiferromagnet heterostructure. Unlike ferromagnetic heterostructures where the Dirac cone is gapped due to time-reversal symmetry breaking, here the Dirac cone is preserved. We demonstrate the existence of a staggered spin density corresponding to a dampinglike torque which is quite robust against scalar impurities when the transport energy is such that the transport is confined to the topological insulator surface. We show the contribution to the nonequilibrium spin density due to both surface and bulk topological insulator bands. Finally, we show that the torques in topological insulator/antiferromagnet heterostructures exhibit an angular dependence that is consistent with the standard spin-orbit torque obtained in Rashba system with some additional structure arising from the interfacial coupling.
\end{abstract}

DOI: 10.1103/PhysRevB.100.014412

\section{INTRODUCTION}

Spin-orbit torque (SOT) has recently become a viable candidate mechanism for the development of magnetic memory devices [1-3]. A typical SOT device mainly consists of two elements: a source of strong spin-orbit coupling (SOC) and a magnetic material $[4,5]$. When a charge current is passed through the material with strong SOC, it produces a nonequilibrium spin density which is utilized to manipulate the magnetic order [6]. In recent years, the field of SOT devices has been revolutionized by two major breakthroughs: one is the discovery of topological insulators (TIs) $[7,8]$ and the other is the rebirth of antiferromagnetic spintronics [9-12]. The strong interfacial SOC in TIs provides a high charge-spin conversion efficiency and thus can be used as an efficient source of SOTs [13-16]. Recent experiments show that TI-based SOT devices can be operated with current densities of the order of $10^{5} \mathrm{~A} / \mathrm{cm}^{2}$ even at room temperature [17-19], which is two orders of magnitude smaller than the switching current density required to operate heavy metal-based SOT devices. Antiferromagnetic materials (AFs), on the other hand, have been known for decades and used extensively as passive exchange bias layers in spintronics spin valves [20]. Ten years ago, it was proposed that the antiferromagnetic order parameter could be manipulated electrically using spin transfer torque $[9,21]$. The recent prediction that SOT could be used to control collinear antiferromagnets [22-25], confirmed shortly after in CuMnAs, opens appealing avenues as antiferromagnets are immune to external magnetic fields and host ultrafast (TeraHertz) dynamics [26,27]. To date, SOT-driven switching has been observed in the noncentrosymmetric CuMnAs [28] and $\mathrm{Mn}_{2} \mathrm{Au}$ crystals [29,30], but also in $\mathrm{Pt} / \mathrm{NiO}$ [31] and

\footnotetext{
*sumit.ghosh@kaust.edu.sa

†aurelien.manchon@kaust.edu.sa
}

compensated $\mathrm{Pt} / \mathrm{CoGd}$ bilayers [32]. The switching is not only robust against an external magnetic field but takes place at much faster rate compared to the ferromagnet-based SOT devices [33-35].

Due to their individual strength, these two fields of research have stimulated a substantial amount of theoretical and experimental research. Considering the promises born by these two classes of materials, it is natural to question the nature of SOTs in AF/TI bilayers and investigate its main features. One major difficulty in this regard is to determine the right material combination, as interfacing TIs with transition metal layers is known to significantly affect the topological surface states (e.g., Refs. [36,37]). Nonetheless, recent experimental progress has been achieved towards the fabrication of AF/TI heterostructures, revealing complex magnetic configurations at the interface and suggesting viable routes towards the observation of SOTs $[38,39]$. A proper theoretical understanding of these systems is therefore highly solicited.

In this work we present a systematic study of spin transport in $\mathrm{AF} / \mathrm{TI}$ heterostructures based on a tight-binding model. We calculate the nonequilibrium spin density using linear response theory and investigate its behavior with respect to the different system's parameters. We show the existence of a nonequilibrium staggered spin density, localized near the interface and immune to scalar impurities at low energy. A similar behavior is observed for the longitudinal conductivity at an energy close to the Dirac point of the TI. The nonequilibrium spin densities show an angular dependence similar to that of a Rashba ferromagnet which can be distorted by the interfacial AF/TI coupling.

\section{METHOD}

We use a tight-binding model to describe the AF/TI heterostructure. The TI is modeled following the method described in Ref. [40] with the same set of parameters. 
(a)

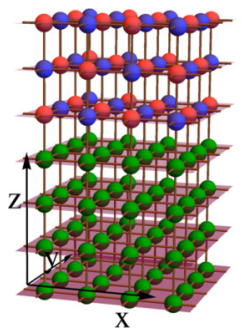

(b)
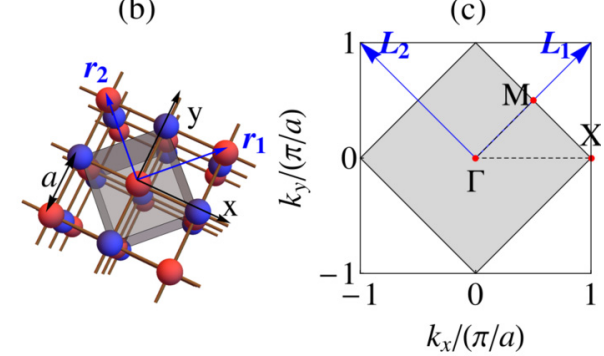

FIG. 1. (a) Schematic of an AF/TI heterostructure. Red and blue spheres denote up and down magnetic moments of the AF layer and the green spheres denote the TI. (b) Top view of an AF lattice. The gray boundary denotes the unit cell. $a$ is the interatomic distance. (c) Corresponding first Brillouin zone (gray region) with lattice vectors $\boldsymbol{L}_{1}$ and $\boldsymbol{L}_{2}$ and high-symmetric points $M, \Gamma$, and $X$ for the $\mathrm{AF} / \mathrm{TI}$ heterostructure.

Although the Hamiltonian is designed on a cubic lattice, near $\Gamma$ point, it is identical to the $\boldsymbol{k} \cdot \boldsymbol{p}$ Hamiltonian of topological insulators like $\mathrm{Bi}_{2} \mathrm{Se}_{3}$ with a hexagonal symmetry [41]. To define the $G$-type antiferromagnet, we have to double the unit cell [Fig. 1(b)] and define lattice vectors $\boldsymbol{r}_{\boldsymbol{1}}=\left(\boldsymbol{e}_{\boldsymbol{x}}+\boldsymbol{e}_{\boldsymbol{y}}\right)$ and $\boldsymbol{r}_{\mathbf{2}}=\left(\boldsymbol{e}_{\boldsymbol{x}}-\boldsymbol{e}_{y}\right)$, where $\boldsymbol{e}_{x}$ and $\boldsymbol{e}_{y}$ are the unit vectors along the $x$ and $y$ axes. The corresponding first Brillouin zone and reciprocal lattice vectors $\left(\boldsymbol{L}_{1}, \boldsymbol{L}_{2}\right)$ are shown in Fig. 1(c).

The Hamiltonian for the TI layers can be written as

$$
H_{\mathrm{TI}}=\sum_{i} a_{i}^{\dagger}\left(h_{0}\right) a_{i}+\sum_{\langle i, j\rangle} a_{i}^{\dagger}\left(h_{i j}\right) a_{j},
$$

where $a_{i}^{\dagger}$ and $a_{i}$ are the creation and annihilation operators for the TI at the $i$ th site, $\langle\cdots\rangle$ denotes the summation over the nearest neighbors, and $h_{0}$ and $h_{i j}$ denote the on-site and hopping elements. For a TI, the on-site and hopping elements are $4 \times 4$ matrices defined as

$$
\begin{aligned}
h_{0} & =\Gamma_{1} M+\mathbb{I}_{4} c, \\
h_{-x}^{\dagger} & =h_{x}=\left(-i \Gamma_{2} A-\Gamma_{1} B-\mathbb{I}_{4} d\right) / 2, \\
h_{-y}^{\dagger} & =h_{y}=\left(i \Gamma_{3} A-\Gamma_{1} B-\mathbb{I}_{4} d\right) / 2, \\
h_{-z}^{\dagger} & =h_{z}=\left(-i \Gamma_{4} A_{1}-\Gamma_{1} B_{1}\right) / 2,
\end{aligned}
$$

where $\mathbb{I}_{4}$ is the identity matrix of rank 4 , while $M, A, B, c, d$, $A_{1}$, and $B_{1}$ are the material parameters [40,41]. The $\Gamma$ matrices are defined as

$$
\begin{array}{ll}
\Gamma_{1}=\sigma_{1} \otimes \mathbb{I}_{2}, & \Gamma_{2}=\sigma_{3} \otimes \sigma_{2}, \\
\Gamma_{3}=\sigma_{3} \otimes \sigma_{1}, & \Gamma_{4}=\sigma_{2} \otimes \mathbb{I}_{2},
\end{array}
$$

where $\mathbb{I}_{2}$ is the identity matrix of rank 2 and $\sigma$ 's are the Pauli matrices for spin 1/2. Similarly, the Hamiltonian for the AF layers is given by

$$
H_{\mathrm{AF}}=\sum_{i} c_{i}^{\dagger}\left(\Delta_{i} \boldsymbol{m} \cdot \boldsymbol{\sigma}+\varepsilon_{0} \mathbb{I}_{2}\right) c_{i}-\sum_{\langle i, j\rangle} c_{i}^{\dagger}\left(t_{i j} \mathbb{I}_{2}\right) c_{j},
$$

where $c_{i}^{\dagger}$ and $c_{i}$ are the creation and annihilation operators for the AF at the $i$ th site, $\Delta_{i}=\Delta(-1)^{x_{i}+y_{i}+z_{i}}$ is the staggered on-site exchange energy, and $\varepsilon_{0}$ is the uniform on-site energy which can tune the position of the AF bands. In the case of

(a)

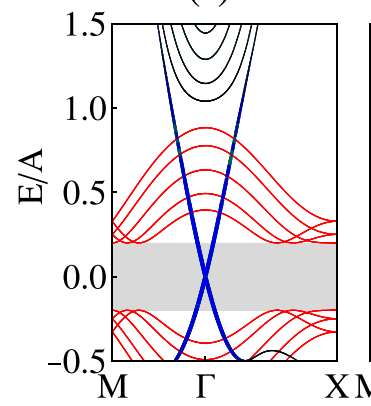

(b)

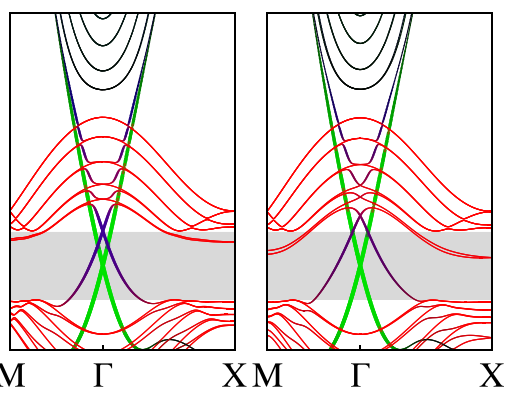

FIG. 2. Band structure of a heterostructure with 10 layers of TI and 5 layers of AF with a coupling strength (a) $t_{C}=0$, (b) $t_{C}=0.5$, and (c) $t_{C}=0.75$. The red, green, blue, and black colors correspond to the contributions from AF layers, bottom TI layer, interfacial TI layer, and bulk TI layers, respectively. The gray region shows the gap due to exchange splitting for the decoupled AF.

AF, we use isotropic real hopping as $t_{x, y, z}=t_{-x,-y,-z}=t_{\mathrm{AF}}$. The TI and AF layers are connected by

$$
H_{\mathrm{AFTI}}=\sum_{\langle i, j\rangle} a_{i}^{\dagger}\left(T_{C}\right) c_{j}+\text { H.c. }, \quad T_{C}=t_{C}\left(\mathbb{I}_{2}, \mathbb{I}_{2}\right),
$$

where H.c. denotes the Hermitian conjugate. The typical parameter values we use here are $A=1.0, B=1.5, M=3.5$, $c=1.5, d=0.75, A_{1}=1.5, B_{1}=1.5, t_{\mathrm{AF}}=0.15$, and $\Delta=$ 0.2 . Unless explicitly mentioned otherwise, we use $\varepsilon=0.0$ and $t_{C}=0.5$ and set the magnetic moments of the AF layer out of plane. We consider a system with 10 layers (20 sites) of TI and 5 layers (10 sites) of AF. The corresponding band structures for different coupling strengths are shown in Fig. 2. Note that the Dirac cone coming from the interfacial TI layer remains gapless [42] and, depending on the $\mathrm{AF} / \mathrm{TI}$ coupling strength, is shifted to slightly higher energy (blue curves). In addition, we notice that the AF gap reduces upon increasing the coupling with the TI surface states.

The nonequilibrium spin density and conductivity are calculated within the linear response framework [40,43-45]. We start by defining the retarded and advanced Green's functions $G^{R, A}(E, \boldsymbol{k})$ at energy $E$ and momentum $\boldsymbol{k}$,

$$
G^{R, A}(E, \boldsymbol{k})=\left[(E \pm i \eta) \mathbb{I}_{n}-H(\boldsymbol{k})\right]^{-1} .
$$

For simplicity, we write it as $G^{R, A}$ in the following section, omitting the explicit dependence of $E$ and $\boldsymbol{k}$. The expectation value of an observable $\mathcal{O}$ due to a perturbation $\mathcal{P}$ consists of two parts:

$$
\left\langle\hat{\mathcal{O}}^{\hat{\mathcal{P}}}\right\rangle=\left\langle\hat{\mathcal{O}}^{\hat{\mathcal{P}}}\right\rangle_{\text {sur }}+\left\langle\hat{\mathcal{O}}^{\hat{\mathcal{P}}}\right\rangle_{\text {sea }},
$$

where $\mathcal{O}_{\text {sur }}$ and $\mathcal{O}_{\text {sea }}$ correspond to contributions from Fermi surface and Fermi sea, respectively, and are defined by

$$
\begin{aligned}
\left\langle\hat{\mathcal{O}}^{\hat{\mathcal{P}}}\right\rangle_{\text {sur }}= & \frac{1}{2 \pi} \int \frac{d^{2} k}{(2 \pi)^{2}} \operatorname{Re}\left\{\operatorname{Tr}\left[\hat{\mathcal{O}} G^{R} \hat{\mathcal{P}}\left(G^{A}-G^{R}\right)\right]\right\}_{E_{F}}, \\
\left\langle\hat{\mathcal{O}}^{\hat{\mathcal{P}}}\right\rangle_{\text {sea }}= & \frac{1}{2 \pi} \int_{-\infty}^{E_{F}} d E \int \frac{d^{2} k}{(2 \pi)^{2}} \\
& \times \operatorname{Re}\left(\operatorname{Tr}\left[\hat{\mathcal{O}} G^{R} \hat{\mathcal{P}} \frac{\partial G^{R}}{\partial E}-\hat{\mathcal{O}} \frac{\partial G^{R}}{\partial E} \hat{\mathcal{P}} G^{R}\right]\right) .
\end{aligned}
$$


(a)
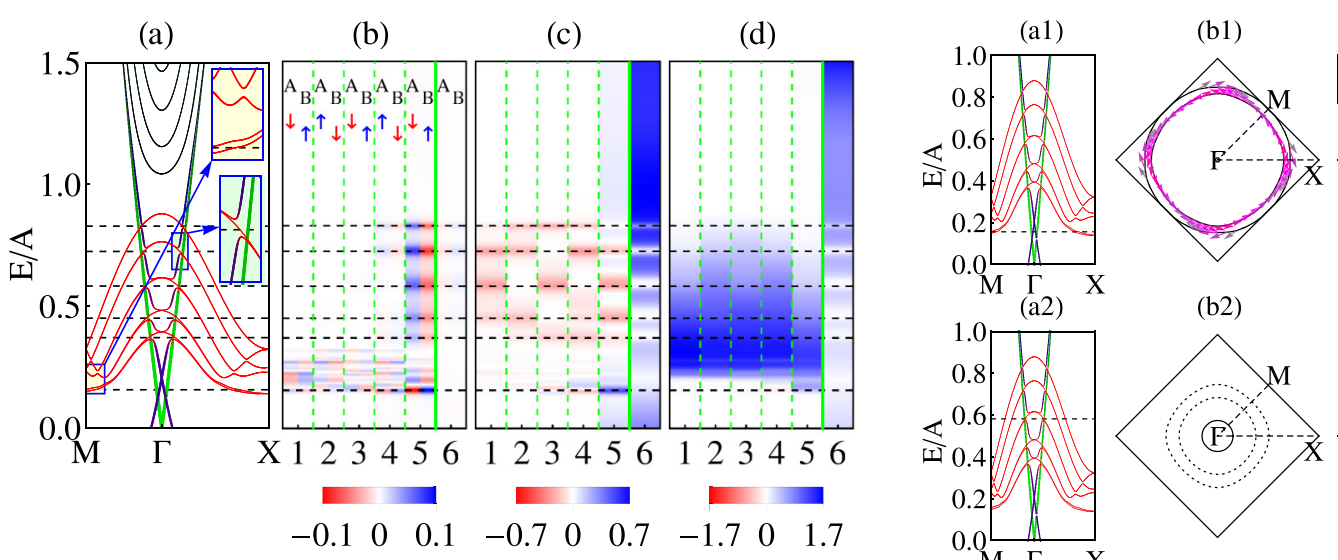

(b2)
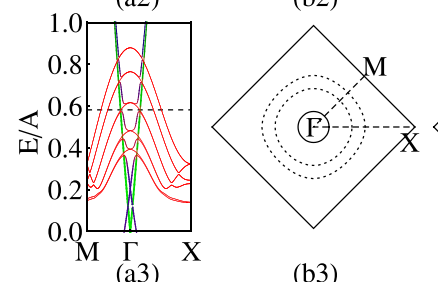

(b3)

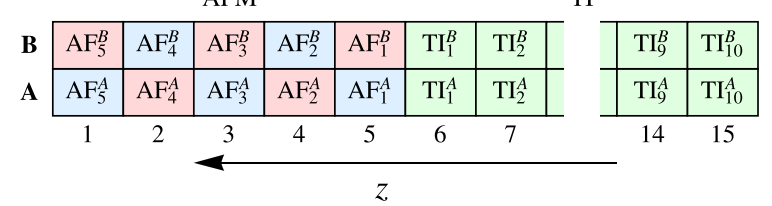

TI

FIG. 3. (a) Band structure, nonequilibrium spin components (b) $S_{x}$, (c) $S_{y}$, and (d) conductivity for sites 1 to 12 with out-of-plane magnetization. The red, blue, green, and black colors in (a) show the contribution from AF, interfacial TI, bottom TI, and bulk TI bands. The horizontal dashed lines denote the peaks of spin densities. In (b), (c), and (d) the vertical dashed green lines show the AF layers and the thick green line indicates the position of the interface. The corresponding configuration is shown in the bottom panel where the blue and red boxes denote AF sites with up and down magnetic moments, and the green boxes correspond to TI sites.

In this article we are interested in the nonequilibrium spin density and conductivity due to an electric field applied along $x$ direction. Therefore the perturbation term is given by $\hat{\mathcal{P}}=e \mathcal{E} v_{x}$, where $v_{x}=\partial H / \partial\left(\hbar k_{x}\right), \mathcal{E}$ is the amplitude of the applied electric field, and $e$ is the electrical charge. Since we are interested in the response function, the computed quantities are normalized with respect to $\mathcal{E}$. The spin operator for individual site is given by

$$
\hat{S}_{i}=\boldsymbol{s} \otimes|i\rangle\langle i|,
$$

where $s=\sigma$ for the AF and $s=\sigma \otimes \mathbb{I}_{2}$ for TI sites. $|i\rangle\langle i|$ is the projection operator for the $i$ th site. Since we are using a bipartite lattice, instead of a site resolved velocity operator, we use an average velocity operator defined as

$$
\hat{v}_{x}^{j}=\left(1 / \hbar\left[\partial H / \partial k_{x}\right] \ldots\left[\operatorname{layer}_{j}\right] \ldots\right) / 2,
$$

where $\cdots\left[\right.$ layer $\left._{j}\right] \cdots$ refers to the fact that we take the block corresponding to the layer containing the $j$ th site from the full matrix. Since each layer contains two sites, we divide this matrix by 2 to obtain the average contribution coming from a single site. The response function for nonequilibrium spin density and the conductivity are given by

$$
\begin{gathered}
\boldsymbol{S}_{i}=\left\langle\hat{\boldsymbol{S}}_{i}^{e \hat{v}_{x}}\right\rangle, \\
\sigma_{x x}^{j}=\left\langle e\left(\hat{v}_{x}^{j}\right)^{e \hat{v}_{x}}\right\rangle .
\end{gathered}
$$

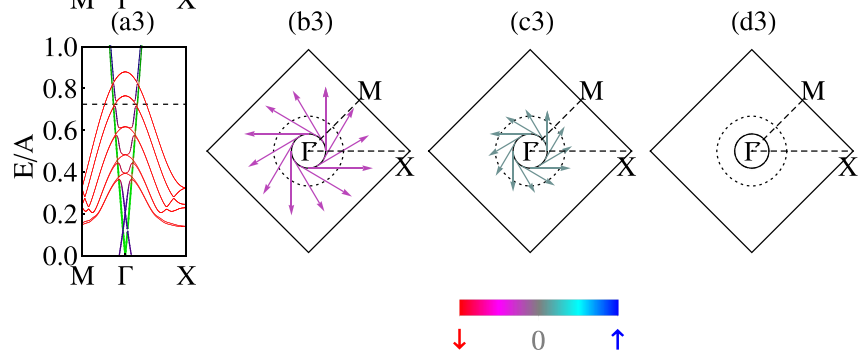

FIG. 4. (a) Band structure and spin texture at site (b) $\mathrm{AF}_{2}^{\mathrm{B}}$, (c) $\mathrm{AF}_{1}^{\mathrm{B}}$, and (d) $\mathrm{TI}_{1}^{\mathrm{B}}$ (see bottom panel in Fig. 3) at different energies [(1) $E / A=0.156$, (2) $E / A=0.582$, (3) $E / A=0.724]$. The arrow indicates the in-plane component and the color of the arrow represents the out-of-plane component. The dotted lines correspond to the degenerate bands.

\section{RESULT AND DISCUSSION}

\section{A. Spatial distribution of the spin density and conductivity}

First, we calculate the site-resolved spin density and conductivity for the AF layer and the interfacial TI layer (Fig. 3). For simplicity, we use natural units, i.e., $e=\hbar=a=1$, which does not affect the qualitative behavior of the observables. From Fig. 2 we see that the coupling between TI and $\mathrm{AF}$ isolates one pair of $\mathrm{AF}$ bands from the conduction bands and brings it lower in the exchange gap region. This pair is dominated by the interfacial AF layer, which is also reflected in the fact that the spin densities and conductivity are stronger in the interfacial layer at this energy. Note that the spin densities show maxima at energies where the coupling between the AF and TI layers are strongest, which breaks the continuity of the TI bands [horizontal dashed lines in Fig. 3(a)]. Correspondingly, the spin densities in the TI layers reduce at these energies. Also note that at lower energy, the spin density has opposite sign. This is because the coupling moves the Dirac cone from the top TI layer higher in energy and since the upper and lower halves of the Dirac cone possess opposite texture, the induced nonequilibrium spin density also switches sign.

To understand the connection between the equilibrium spin texture and nonequilibrium spin density, we calculate the siteresolved spin texture in momentum space at different energies. Figure 4 shows the band structure and the corresponding spin texture for site $8\left(\mathrm{AF}_{2}^{\mathrm{B}}, \downarrow\right), 10\left(\mathrm{AF}_{1}^{\mathrm{B}}, \uparrow\right)$, and $12\left(\mathrm{TI}_{1}^{\mathrm{B}}\right)$ for different energies. At $E / A=0.156$, the degeneracy of the 
(a)

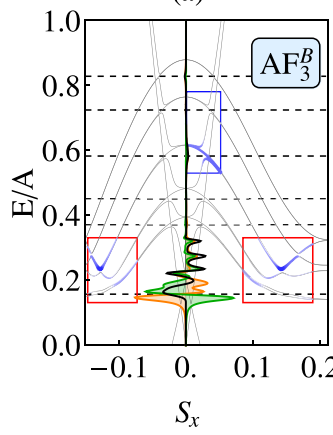

$\square$ Surface (b)

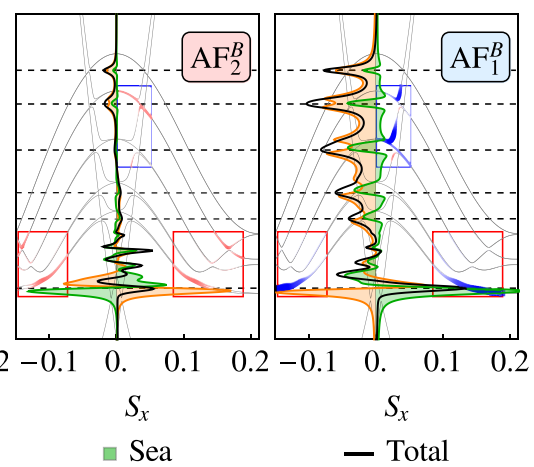

FIG. 5. Contribution from Fermi surface (orange) and Fermi sea (green) for the $B$ sublattice of the first three AF layers. The equilibrium $S_{z}$ component is shown in the boxed regions where red and blue colors correspond to negative and positive values. The maximum value in the red box is 0.35 and the maximum value in the blue box is 0.1 .

AF bands has been lifted due to coupling with the TI layer, which is manifested as two isolated rings in the Brillouin zone. Note that these two rings have opposite textures and, in the case of a degenerate band, they cancel each other. This is why the nonequilibrium $S_{y}$, and therefore $S_{x}$, is zero in most of the regions. We further see that due to confinement effect, the AF states at different energies are dominated by different layers. For example, $E / A=0.582$ is dominated by the tenth site (fifth layer), whereas $E / A=0.724$ is dominated by the eighth site (fourth layer). This explains the variation of the $S_{y}$ component across different layers (Fig. 3). Note that at low energy the AF bands are well separated in momentum from the interfacial TI Dirac cone, which allows the TI layer to induce SOC in the AF layer and also to retain its own texture. From the slope of the band structure, one can see that the TI and the AF layers have opposite group velocity within the intermediate energy range $[0.15<E / A<0.85$, which is roughly the region between the second and sixth horizontal dashed lines in Fig. 3(a)]. As a result, within this regime, the AF layer and the TI layer possess an opposite $S_{y}$ component in spite of having similar spin texture.

\section{B. Fermi surface and sea contributions to $S_{x}$}

The nonequilibrium $S_{x}$ component is particularly important because it enables the electrical control of the AF magnetic order. As a matter of fact, its magnitude depends both on the induced SOC as well as on the magnetization of the AF sublattices. As a result, one can obtain a staggered $S_{x}$ that can be utilized to switch the antiferromagnetic order parameter [22,24]. Interestingly, unlike $S_{y}, S_{x}$ has finite contributions from both Fermi surface [Eq. (8)] and Fermi sea [Eq. (9)] (see Fig. 5), which indicates its topological origin.

One can readily see that both of the sea and surface terms dominate in the region where the degeneracy of the AF bands is lifted. Note that the Fermi sea contribution attains a maximum value in the middle of the gap where the surface contribution shows a minimum. This is because while the Fermi surface term depends on the induced SOC, the Fermi

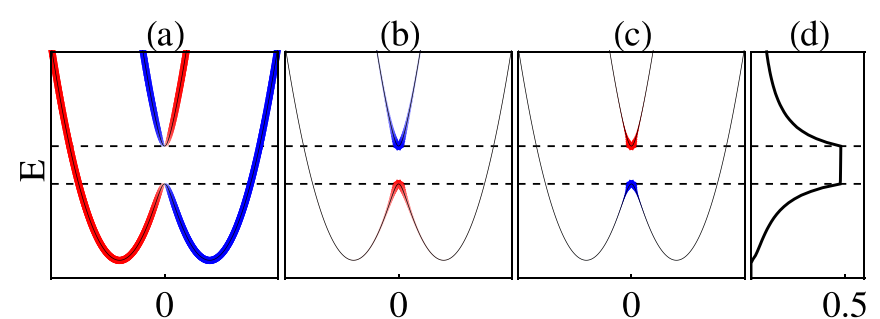

FIG. 6. Band structure of a Rashba ferromagnet, Eq. (14), along $k_{x}$ with projected (a) $S_{y}$, (b) $S_{z}$, (c) Berry curvature, and (d) integrated Berry curvature or anomalous Hall coefficient. The dashed lines show the exchange gap. Here we use $\hbar=1, m_{0}=0.5, \alpha=1$, and $\Delta=0.05$.

sea term depends on the strength of Berry curvature [46], which is maximal at the avoided band crossing points marked in Fig. 5. Indeed, while a pure Dirac cone does not host any Berry flux, its hybridization with the AF bands liberates a Berry curvature at the avoided crossing points. This results in "hot spots" of nonequilibrium spin density in the band structure.

To understand the topological origin of the nonequilibrium $S_{x}$ one needs to study the relevant topological invariant. However, constructing a proper topological invariant for the interfacial states of such an antiferromagnetic heterostructure is mathematically quite challenging and is beyond the scope of the present work. Instead, we present a heuristic argument to determine the Berry curvature contribution. We note that the Berry curvature is analogous to a magnetic field whose strength is maximum when the spin texture encounters a singularity marked by a zero in-plane and finite out-of-plane component. To demonstrate that, we consider the Rashbaferromagnet Hamiltonian

$$
H_{R}=\hbar^{2} k^{2} / 2 m_{0}+\alpha \hat{z} .(\boldsymbol{\sigma} \times \boldsymbol{p})+\Delta \sigma_{z} .
$$

Following Ref. [47], the Berry curvature reads

$$
\Omega^{ \pm}(k)=\mp \frac{1}{2} \frac{\alpha^{2} \Delta}{\left(\Delta^{2}+\alpha^{2} k^{2}\right)^{3 / 2}},
$$

which can readily be compared with the momentumdependent $z$ component of the spin density,

$$
S_{z}^{ \pm}(k)= \pm \frac{\Delta}{\left(\Delta^{2}+\alpha^{2} k^{2}\right)^{1 / 2}},
$$

where \pm corresponds two different bands. It clearly appears that the maximum in Berry curvature coincides with a maximum in $S_{z}$. The corresponding band structure along with its spin projection and Berry curvature is shown in Fig. 6. One can readily see that the Berry curvature is localized in a region where the out-of-plane component of spin is maximum, consistently with Eqs. (15) and (16) above. At these points, the in-plane texture vanishes, creating a singularity. Therefore we track the equilibrium $S_{z}$ as a smoke signal for Berry curvature.

From Fig. 5 we see that most of the equilibrium $S_{z}$ component is localized at the bottom of the positive AF bands and therefore most of the nonequilibrium $S_{x}$ component is also generated in this region. Since the bands with opposite texture have the same $S_{z}$ component [Fig. 4(c1)], the Fermi surface component switches sign as we move from the first AF band 
to the second one $(E / A \sim 0.156)$. The corresponding Berry curvature is localized at the bottom of the bands where the Fermi sea term changes sign. At higher energy $(E / A \sim 0.724)$ opposite textures possess opposite $S_{z}$ (see the blue box in Fig. 5) and therefore the Fermi surface term does not change sign anymore. The Fermi sea term being dependent on the Berry curvature still oscillates with the sign of $S_{z}$. For a decoupled AF band, there is no equilibrium $S_{z}$ component near the band maximum. In an $\mathrm{AF} / \mathrm{TI}$ heterostructure, this component, and hence its Berry curvature, is produced by the interaction between the TI and the AF, and is therefore localized close to the interface. As a result, for $E / A>0.3$, the nonequilibrium $S_{x}$ component is finite only near the interface [Fig. 3(b)] although the $S_{y}$ component is visible deep inside [Fig. 3(c)]. This is also reflected in the fact that the Fermi sea term dominates in the bottom of the positive AF band for the first $A F$ layer $\left[\mathrm{AF}_{1}^{\mathrm{B}}\right.$, Fig. 5(c)], whereas for $\mathrm{AF}_{2}^{\mathrm{B}}$ onwards, the surface term dominates. As a result, while in $\mathrm{AF}_{1}^{\mathrm{B}}$ the $S_{x}$ component has the same sign as the local magnetic moment, $\mathrm{AF}_{2}^{\mathrm{B}}$ and $\mathrm{AF}_{3}^{\mathrm{B}}$ have opposite signs.

\section{Effect of AF/TI coupling}

To understand the cumulative behavior, we introduce the staggered $x$ component of the spin density $S_{x}^{\text {stg }}$, the total $y$ component $S_{y}^{\text {tot }}$, and the total conductivity $\sigma_{x x}^{\text {tot }}$, defined as

$$
\begin{gathered}
S_{x}^{\mathrm{stg}}=\sum_{i}^{\uparrow} S_{x}^{i}-\sum_{j}^{\downarrow} S_{x}^{j}, \\
S_{y}^{\mathrm{tot}}=\sum_{i}^{\uparrow} S_{y}^{i}+\sum_{j}^{\downarrow} S_{y}^{j}, \\
\sigma_{x x}^{\mathrm{tot}}=\sum_{i}^{\uparrow} \sigma_{x x}^{j}+\sum_{j}^{\downarrow} \sigma_{x x}^{i},
\end{gathered}
$$

where the indices $i$ and $j$ run over the AF sites with up and down spin. The $S_{x}^{\text {stg }}$ component induces the dampinglike torque that enables the electrical control of the Néel order parameter [21,22,24], while the $S_{y}^{\text {tot }}$ induces an effective field that does not contribute to the switching. First we look at the effect of the coupling between the AF and the TI, which is the only way to imprint SOC on the AF layer and produces the nonequilibrium spin density.

Figure 7 shows the variation of $S_{x}^{\text {stg }}, S_{y}^{\text {tot }}$, and $\sigma_{x x}^{\text {tot }}$ as a function of energy and with respect to the coupling strength between the AF and TI layers $\left(t_{C}\right)$. For better understanding, we divide the energy range into three regions. Region I corresponds to the exchange gap of the decoupled AF bands. This region can be occupied by an AF band only upon turning on the coupling between AF and TI (Fig. 2). Region II spans the energy range corresponding to the bottom of the positive AF bands, which corresponds to the maximum density of states and also contains strong equilibrium $S_{z}$ component. The coupling opens a gap between different bands in this region (Fig. 2). Region III contains all the energies above region II. The coupling mainly distorts the TI bands rather than the AF bands here.

$S_{y}^{\text {tot }}$ increases in both region I and region II as it depends only on the induced SOC. Note that the $S_{x}$ component
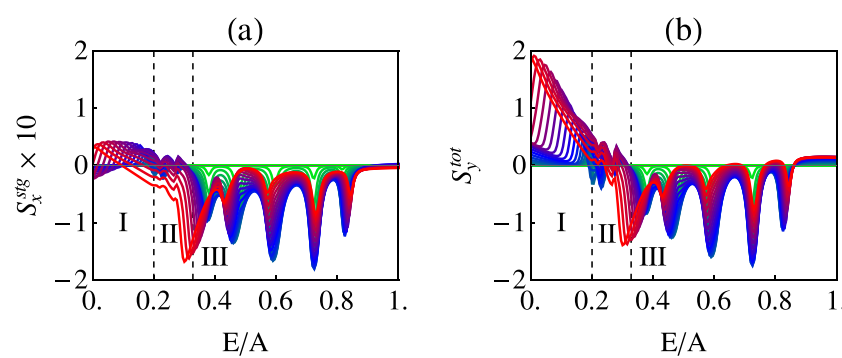

(c)
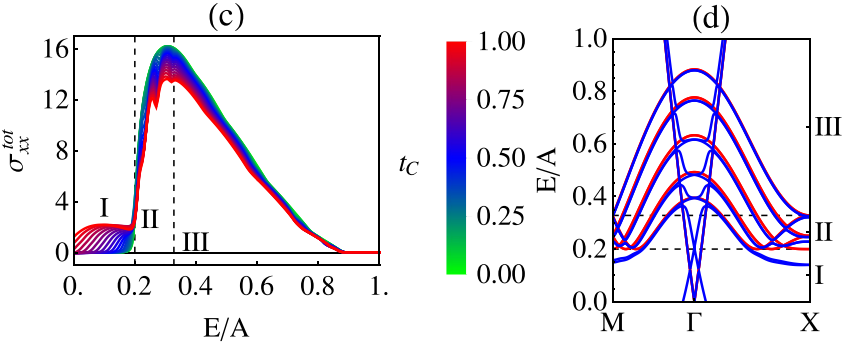

FIG. 7. (a) $S_{x}^{\text {stg }}$, (b) $S_{y}^{\text {tot }}$, and (c) $\sigma_{x x}^{\text {tot }}$ of the AF layer as a function of the coupling strength whose value is shown in the color bar. (d) The band structure of AF/TI with $t_{C}=0.5 \mathrm{~A}$ (blue) and $t_{C}=0$ (red). The dashed lines show three different regions I, II, and III.

switches sign with respect to the local magnetization in the first two consecutive layers (Fig. 3), which reduces $S_{x}^{\text {stg }}$. This effect is more prominent in region I as it is dominated both by the first $\left(\mathrm{AF}_{1}^{\mathrm{A}, \mathrm{B}}\right)$ and second $\left(\mathrm{AF}_{2}^{\mathrm{A}, \mathrm{B}}\right)$ interfacial as well as bulk AF layers (Fig. 3). This is also reflected in the equilibrium spin texture (Fig. 4). As a result, in region I, $S_{x}^{\text {stg }}$ starts decreasing for stronger coupling strength [Fig. 7(a)]. Region II is less dominated by the interfacial AF layer and, therefore, the cancellation due to opposite texture is minimized. Moreover, stronger coupling opens larger gaps, which provides more $S_{z}$ component (i.e., stronger Berry curvature) giving rise to larger value of $S_{x}^{\text {stg }}$.

In region III, the coupling breaks the degeneracy of the AF bands, which ensures that only one type of texture dominates. For smaller coupling, the two opposite textures have almost the same contribution and cancel each other. For stronger coupling, the TI bands undergo more distortion resulting in reduced SOC and weak spin texture. Therefore we see that in region III both $S_{y}^{\text {tot }}$ and $S_{x}^{\text {stg }}$ attain a maximum value for intermediate coupling strength. The conductivity on the other hand depends on the density of states. It increases in region I and decreases in regions II and III with the coupling strength, as the coupling pulls down the AF states from regions II and III to region I (Fig. 7).

\section{Coupling to surface versus bulk TI states}

So far we have considered the AF bands coupled to the surface TI bands only. In reality the AF bands can be connected to the bulk TI bands as well, or can be coupled to both surface and bulk. To understand these different scenarios, we change the position of the AF bands by varying the on-site energy $\left[\varepsilon_{0}\right.$, Eq. (4)] of the AF layer.

Figure 8 shows the variation of $S_{x}^{\text {stg }}, S_{y}^{\text {tot }}$, and $\sigma_{x x}^{\text {tot }}$ for different on-site energies $\varepsilon_{0}$. The total conductivity $\left(\sigma_{x x}^{\text {tot }}\right)$ is confined within the region marked by the dashed line denoting 
(a)

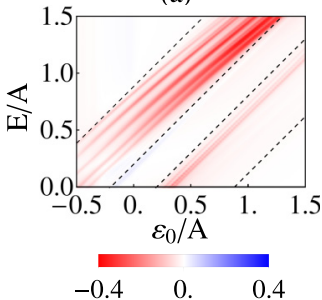

(b)

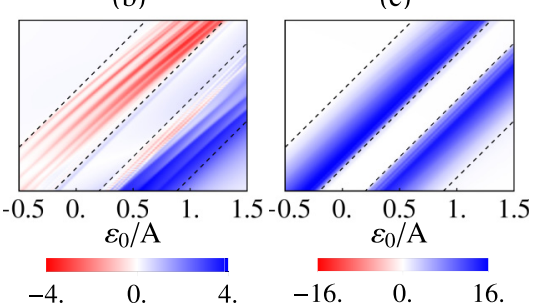

FIG. 8. Energy dependence of (a) $S_{x}^{\text {stg }}$, (b) $S_{y}^{\text {tot }}$, and (c) $\sigma_{x x}^{\text {tot }}$ of the $\mathrm{AF}$ layer as a function of the on-site energy of the AF layer with $t_{C}=0.5 A$. The inclined dashed line shows the region spanned by the decoupled AF bands.

the energy range occupied by the AF bands. For $S_{x}^{\text {stg }}$ and $S_{y}^{\text {tot }}$, one can clearly see five branches corresponding to the intersections of AF bands with the TI bands at lower energies. Careful observation reveals a faint branch with opposite sign within the exchange gap region which corresponds to the interfacial $\mathrm{AF}$ band. For larger values of $\varepsilon_{0}$, the $\mathrm{AF}$ bands are coupled to more bulk TI bands, which increases both $S_{y}$ and $S_{x}$. However, one should note that in this case more current flows through the bulk TI, which effectively reduces the spin Hall angle and hence the efficiency of the system [40]. Besides, the bulk bands are not immune to scattering, which makes the coupling to the bulk bands inefficient against impurity scattering.

\section{E. Effect of impurities}

Next, we study the effect of impurity scattering modeled as a constant broadening, an approach that is found to be sufficient in realistic systems as well [43]. Figure 9 shows the variation of $S_{x}^{\text {stg }}, S_{y}^{\text {tot }}$, and $\sigma_{x x}^{\text {tot }}$ with the broadening parameter $(\eta)$. We have already seen that the nonequilibrium $S_{x}$ and $S_{y}$ are maximum only at some specific values of energies (Figs. 3

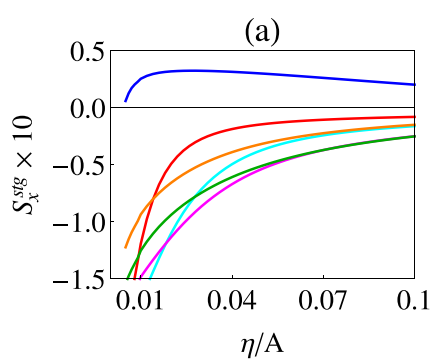

(c)

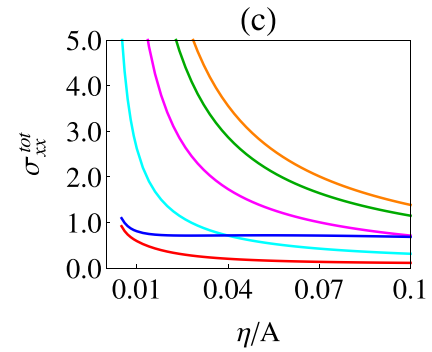

FIG. 9. (a) $S_{x}^{\text {stg }}$, (b) $S_{y}^{\text {tot }}$, and (c) $\sigma_{x x}^{\text {tot }}$ as a function of broadening. Different line colors correspond to different energies as shown in (d) along with the band structure. and 7). Here we consider the energies marked by the dashed line in Figs. 3 and 5.

From Fig. 9 we see that for energies coming from region III (Fig. 7), $S_{x}^{\text {stg }}, S_{y}^{\text {tot }}$, and $\sigma_{x x}^{\text {tot }}$ show a $1 / \eta$ decay which is the characteristic of a metallic system. This behavior is expected for $S_{y}^{\text {tot }}$ and $\sigma_{x x}^{\text {tot }}$, which are dominated by Fermi surface contributions and are therefore extrinsic (impurity dependent). However, it is quite surprising for $S_{x}^{\text {stg }}$. Indeed, previous works have shown that the dampinglike torque is associated with the intrinsic Berry curvature in mixed spinmomentum space, and is thereby constant in the weak disorder limit $[22,23,43,44,46]$. This $1 / \eta$ dependence of the $S_{x}^{\text {stg }}$ component is also in sharp contrast with our previous work on TI/ferromagnet heterostructures (see Fig. 9 in Ref. [40]). This dependence can be understood by noticing that the dampinglike torque (i.e., $S_{x}^{\text {stg }}$ ) is only expected to be constant in the weak disorder limit, i.e., as long as the broadening $\eta$ is smaller than the energy difference between neighboring bands [44]. In Fig. 9 we selected transport energies corresponding to cases where the hybridization between the TI and AF bands is maximized, and therefore the above condition on $\eta$ is not satisfied for the disorder strength considered, resulting in the $1 / \eta$ behavior.

Notice that the $\eta$ dependence of $S_{x}^{\text {stg }}, S_{y}^{\text {tot }}$, and $\sigma_{x x}^{\text {tot }}$ is completely different when the transport energy lies in region I (see Fig. 9, blue line). We see that $\sigma_{x x}^{\text {tot }}$ remains constant as the effect of the impurities is quenched by the strong spin polarization. $S_{y}^{\text {tot }}$ also falls down but with a much slower rate, which indicates the weakening of the induced SOC. $S_{x}^{\text {stg }}$ shows an initial increase and then becomes almost constant, which also points towards its topologically protected origin. The enhancement of $S_{x}^{\text {stg }}$ component due to scattering has also been observed in two-dimensional antiferromagnetic TIs [48] as well as TI/ferromagnet heterostructures [40].

\section{F. Angular dependence of torques}

Finally, we present the angular dependence of the fieldlike and dampinglike torques with respect to the polar $(\theta)$ and azimuthal $(\phi)$ angles for different planes. The results are reported in Fig. 10. Note that in the case of out-of-plane magnetization the fieldlike and dampinglike torques are directly proportional to $S_{y}^{\text {tot }}$ and $S_{x}^{\text {stg }}$. Therefore, one can easily understand the behavior of the fieldlike and dampinglike torques from the $S_{x}^{\text {stg }}$ and $S_{y}^{\text {tot }}$ components. In general the torque components at each site can be expressed as

$$
\begin{aligned}
\boldsymbol{T}^{i} & =\boldsymbol{m}^{i} \times \boldsymbol{S}^{i} \\
& =\tau_{F}^{i}\left(\boldsymbol{m}^{i} \times \boldsymbol{e}_{\boldsymbol{y}}\right)+\tau_{D}^{i} \boldsymbol{m}^{i} \times\left(\boldsymbol{m}^{i} \times \boldsymbol{e}_{\boldsymbol{y}}\right) .
\end{aligned}
$$

$\boldsymbol{e}_{\boldsymbol{x}}$ is the direction of the current flow and $\boldsymbol{e}_{z}$ is normal to the surface. The general expressions for $\tau_{F, D}^{i}$ for any arbitrary angle are given by

$$
\begin{aligned}
\tau_{F}^{i}= & \left(\boldsymbol{m}^{i} \times \boldsymbol{S}^{i}\right) \cdot\left(\boldsymbol{m}^{i} \times \boldsymbol{e}_{\boldsymbol{y}}\right) /\left|\boldsymbol{m}^{i} \times \boldsymbol{e}_{\boldsymbol{y}}\right|^{2} \\
= & -\frac{\sin \phi \cos \phi \sin ^{2} \theta}{1-\sin ^{2} \phi \sin ^{2} \theta} S_{x}^{i}+S_{y}^{i} \\
& -\frac{\sin \phi \sin \theta \cos \theta}{1-\sin ^{2} \phi \sin ^{2} \theta} S_{z}^{i},
\end{aligned}
$$


(a)

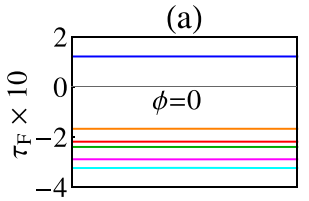

(d)

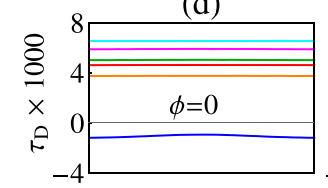

(g)
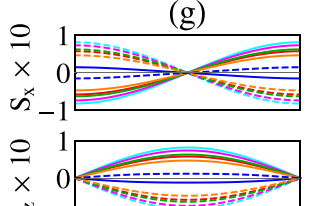

N-1

ஸे -1

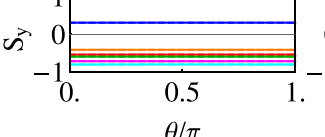

$\theta / \pi$ (b)

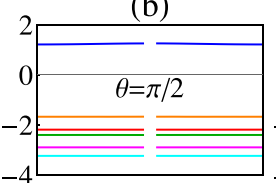

(e)

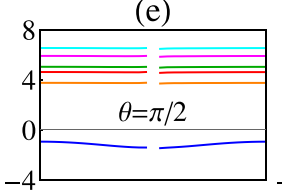

(h)

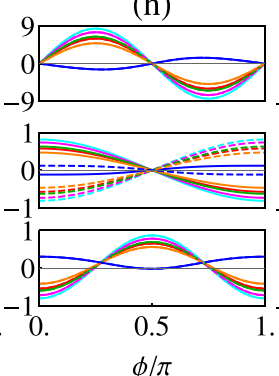

$\phi / \pi$ (c)

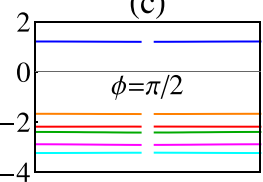

(f)

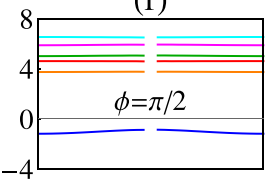

(i)

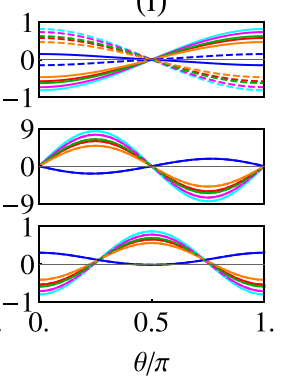

FIG. 10. Variation of (a)-(c) $\tau_{F L}$ and (d)-(f) $\tau_{D L}$ with respect to $\theta[(\mathrm{a})$ and (c) and (d) and (f)] and $\phi[(\mathrm{b})$ and (e)]. The inset value corresponds to the fixed angle. (g), (h), and (i) The $S_{x}, S_{z}$, and $S_{y}$ components, where solid and dashed lines denote the contribution from AF sites with up and down spin. The colors represent different energies as described in Fig. 9(d).

$$
\begin{aligned}
\tau_{D}^{i} & =\left(\boldsymbol{m}^{i} \times \boldsymbol{S}^{i}\right) \cdot\left[\boldsymbol{m}^{i} \times\left(\boldsymbol{m}^{i} \times \boldsymbol{e}_{\boldsymbol{y}}\right)\right] /\left|\boldsymbol{m}^{i} \times\left(\boldsymbol{m}^{i} \times \boldsymbol{e}_{\boldsymbol{y}}\right)\right|^{2} \\
& =-\frac{\cos \theta}{1-\sin ^{2} \phi \sin ^{2} \theta} S_{x}^{i}+\frac{\sin \theta \cos \phi}{1-\sin ^{2} \phi \sin ^{2} \theta} S_{z}^{i} .
\end{aligned}
$$

Note that each layer contains two sites with opposite magnetization. Since these two sites have the same sign of induced SOC, they have the same sign of fieldlike coefficient $\left(\tau_{F}\right)$ and the opposite sign of dampinglike coefficient $\left(\tau_{D}\right)$. Therefore, we define the total fieldlike and dampinglike torque coefficients as

$$
\begin{aligned}
& \tau_{F}=\sum_{i}^{\uparrow} \tau_{F}^{i}+\sum_{j}^{\downarrow} \tau_{F}^{j}, \\
& \tau_{D}=\sum_{i}^{\uparrow} \tau_{D}^{i}-\sum_{j}^{\downarrow} \tau_{D}^{j} .
\end{aligned}
$$

From Fig. 10 one can readily see that both $\tau_{F}$ and $\tau_{D}$ do not show any angular dependence for all energies dominated by the bulk AF layers (regions II and III in Fig. 7). When the magnetization is in the $x z$ plane [Figs. 10(a) and 10(d)], it does not affect the nonequilibrium $S_{y}$ component. As a

TABLE I. Torque coefficients $\tau_{F}^{i}$ and $\tau_{D}^{i}$ for $z x, x y$, and $y z$ plane.

\begin{tabular}{lcc}
\hline \hline Plane & $\tau_{F}^{i}$ & $\tau_{D}^{i}$ \\
\hline$z x(\phi=0)$ & $S_{y}^{i}$ & $-S_{x}^{i} \cos \theta+S_{z}^{i} \sin \theta$ \\
$x y(\theta=\pi / 2)$ & $S_{y}^{i}-S_{x}^{i} \tan \phi$ & $S_{z}^{i} / \cos \phi$ \\
$x y(\phi=\pi / 2)$ & $S_{y}^{i}-S_{z}^{i} \tan \theta$ & $S_{x}^{i} / \cos \theta$ \\
\hline \hline
\end{tabular}

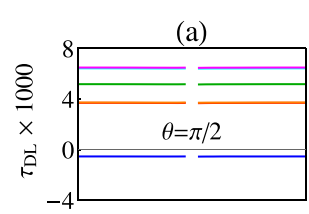

(d)
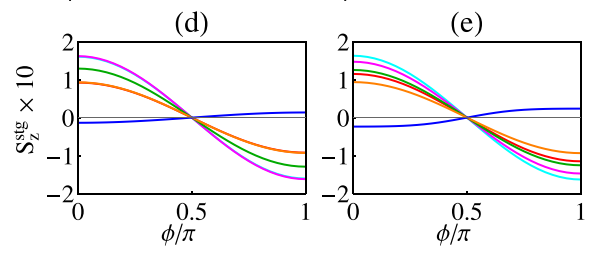

(b)

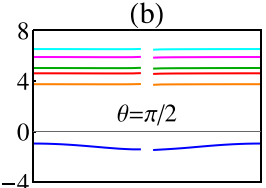

(e)

FIG. 11. Variation of $\tau_{D}$ in the $x y$ plane for (a) $t_{C}=0.25$, (b) $t_{C}=$ 0.50 , and (c) $t_{C}=0.75$ and corresponding nonequilibrium $S_{z}(\mathrm{~d})-(\mathrm{f})$. Different colors correspond to the energies at which $S_{z}$ has peaks. The band structures are shown in Fig. 2.

result, $\tau_{F}$ remains constant for all energies. Since $S_{x, z}$ is generated through the interaction with the $z, x$ components of the magnetization, they change as $\cos \theta, \sin \theta$, respectively, and therefore $\tau_{D}$ remains constant. The situation is different for the $x y$ and $y z$ planes. In these cases, the nonequilibrium $S_{y}$ component also has a contribution from the $y$ component of the magnetization. In addition, the texture itself is modified resulting in a complex angular dependence. Note that for the $x y, y z$ planes, the periodicity of $S_{y}$ and $S_{x, z}$ seems to be doubled compared to the periodicity of $S_{z, x}$. Careful observation shows that the variation is not strictly sinusoidal and depends on the strength of the induced SOC. As a result, the blue lines undergo less distortion due to their stronger SOC compared to the other lines. The $S_{z, x}$ component, on the other hand, is generated through the interaction with the $x, z$ components of the magnetization and therefore shows a $\cos \phi, \theta$ dependence. Note that in the case of $\tau_{D}$, there is small deviation from constant value for the blue line in all three cases. This effect comes from the dependence of the band structure on the magnetization direction, due to its interplay with SOC [23]. For $\tau_{F}$ this effect is nullified by the contribution from $S_{y}$ and $S_{x, z}$. This is further clarified by showing the angular dependence of $\tau_{D}$ and $S_{z}$ in the $x y$ plane for different coupling strengths (Fig. 11).

\section{CONCLUSION}

In this article we present a systematic study of the nonequilibrium spin density and SOT for an AF/TI heterostructure using a tight-binding model within the framework of linear response theory. From the site-resolved spin density, we show the existence of a staggered $S_{x}$ component that can be utilized to switch the antiferromagnetic order parameter. We explain the behavior of the different spin components at different energies and show their connections with the band structure and equilibrium spin texture. We show that for an out-ofplane magnetization, the $S_{x}$ component possesses both Fermi sea and Fermi surface contributions. The contribution from the Fermi sea points towards its topological origin, which is later verified from the impact of impurities. We further study the effect of the coupling between the AF and TI layers and show that the $S_{x}$ component attains its maximum value for an intermediate coupling strength, whereas the $S_{y}$ 
component shows different behavior at different energies. The nonequilibrium spin density also depends on the total amount of overlap, which we demonstrate by coupling the AF bands with both the surface and the bulk TI bands. However, the spin density produced away from the Dirac cone of the TI is not robust against impurities and falls down rapidly with growing impurity strength. Interestingly we find that near the Dirac cone, the $S_{x}$ component is slightly enhanced by the impurities. At this energy, the conductivity is not affected by the impurities. Finally we show the angular dependence of the nonequilibrium spin densities as well as the different torque coefficients, which display the same behavior as the two-dimensional Rashba gas for the bulk AF bands [23]. However within the AF gap, the dampinglike torque shows a complex angular dependence due to the distortion of band structure, which can be enhanced with the coupling strength between AF and TI.

\section{ACKNOWLEDGMENTS}

The authors acknowledge computing time on the supercomputer SHAHEEN at KAUST Supercomputing Center and the team assistance. This work is supported by the King Abdullah University of Science and Technology (KAUST).
[1] A. D. Kent and D. C. Worledge, Nat. Nanotechnol. 10, 187 (2015).

[2] S.-W. Lee and K.-J. Lee, Proc. IEEE 104, 1831 (2016).

[3] N. Sato, F. Xue, R. M. White, C. Bi, and S. X. Wang, Nat. Electron. 1, 508 (2018).

[4] I. M. Miron, K. Garello, G. Gaudin, P.-J. Zermatten, M. V. Costache, S. Auffret, S. Bandiera, B. Rodmacq, A. Schuhl, and P. Gambardella, Nature (London) 476, 189 (2011).

[5] L. Liu, C.-F. Pai, Y. Li, H. W. Tseng, D. C. Ralph, and R. A. Buhrman, Science 336, 555 (2012).

[6] A. Manchon, J. Zelezný, I. M. Miron, T. Jungwirth, J. Sinova, A. Thiaville, K. Garello, and P. Gambardella, arXiv:1801.09636.

[7] M. Z. Hasan and C. L. Kane, Rev. Mod. Phys. 82, 3045 (2010).

[8] X.-L. Qi and S.-C. Zhang, Rev. Mod. Phys. 83, 1057 (2011).

[9] A. S. Núñez, R. A. Duine, P. Haney, and A. H. MacDonald, Phys. Rev. B 73, 214426 (2006).

[10] E. V. Gomonay and V. M. Loktev, Low Temp. Phys. 40, 17 (2014).

[11] T. Jungwirth, X. Marti, P. Wadley, and J. Wunderlich, Nat. Nanotechnol. 11, 231 (2016).

[12] V. Baltz, A. Manchon, M. Tsoi, T. Moriyama, T. Ono, and Y. Tserkovnyak, Rev. Mod. Phys. 90, 015005 (2018).

[13] A. R. Mellnik, J. S. Lee, A. Richardella, J. L. Grab, P. J. Mintun, M. H. Fischer, A. Vaezi, A. Manchon, E.-A. Kim, N. Samarth, and D. C. Ralph, Nature (London) 511, 449 (2014).

[14] Y. Fan, P. Upadhyaya, X. Kou, M. Lang, S. Takei, Z. Wang, J. Tang, L. He, L.-t. Chang, M. Montazeri, G. Yu, W. Jiang, T. Nie, R. N. Schwartz, Y. Tserkovnyak, and K. L. Wang, Nat. Mater. 13, 699 (2014).

[15] Y. Wang, P. Deorani, K. Banerjee, N. Koirala, M. Brahlek, S. Oh, and H. Yang, Phys. Rev. Lett. 114, 257202 (2015).

[16] Y. Fan, X. Kou, P. Upadhyaya, Q. Shao, L. Pan, M. Lang, X. Che, J. Tang, M. Montazeri, K. Murata, L.-T. Chang, M. Akyol, G. Yu, T. Nie, K. L. Wong, J. Liu, Y. Wang, Y. Tserkovnyak, and K. L. Wang, Nat. Nanotechnol. 11, 352 (2016).

[17] J. Han, A. Richardella, S. A. Siddiqui, J. Finley, N. Samarth, and L. Liu, Phys. Rev. Lett. 119, 077702 (2017).

[18] Y. Wang, D. Zhu, Y. Wu, Y. Yang, J. Yu, R. Ramaswamy, R. Mishra, S. Shi, M. Elyasi, K.-L. Teo, Y. Wu, and H. Yang, Nat. Commun. 8, 1364 (2017).
[19] M. DC, R. Grassi, J.-Y. Chen, M. Jamali, D. Reifsnyder Hickey, D. Zhang, Z. Zhao, H. Li, P. Quarterman, Y. Lv, M. Li, A. Manchon, K. A. Mkhoyan, T. Low, and J.-P. Wang, Nat. Mater. 17, 800 (2018).

[20] J. Nogués and I. K. Schuller, J. Magn. Magn. Mater. 192, 203 (1999).

[21] H. V. Gomonay and V. M. Loktev, Phys. Rev. B 81, 144427 (2010).

[22] J. Železný, H. Gao, K. Výborný, J. Zemen, J. Mašek, A. Manchon, J. Wunderlich, J. Sinova, and T. Jungwirth, Phys. Rev. Lett. 113, 157201 (2014).

[23] J. Železný, H. Gao, A. Manchon, F. Freimuth, Y. Mokrousov, J. Zemen, J. Mašek, J. Sinova, and T. Jungwirth, Phys. Rev. B 95, 014403 (2017).

[24] A. Manchon, J. Phys.: Condens. Matter 29, 104002 (2017).

[25] H. Watanabe and Y. Yanase, Phys. Rev. B 98, 220412(R) (2018).

[26] R. Cheng, M. W. Daniels, J.-G. Zhu, and D. Xiao, Phys. Rev. B 91, 064423 (2015).

[27] R. Cheng, D. Xiao, and A. Brataas, Phys. Rev. Lett. 116, 207603 (2016).

[28] P. Wadley, B. Howells, J. Železný, C. Andrews, V. Hills, R. P. Campion, V. Novak, K. Olejnik, F. Maccherozzi, S. S. Dhesi, S. Y. Martin, T. Wagner, J. Wunderlich, F. Freimuth, Y. Mokrousov, J. Kune, J. S. Chauhan, M. J. Grzybowski, A. W. Rushforth, K. W. Edmonds, B. L. Gallagher, and T. Jungwirth, Science 351, 587 (2016).

[29] S. Y. Bodnar, L. Šmejkal, I. Turek, T. Jungwirth, O. Gomonay, J. Sinova, A. A. Sapozhnik, H.-J. Elmers, M. Kläui, and M. Jourdan, Nat. Commun. 9, 348 (2018).

[30] M. Meinert, D. Graulich, and T. Matalla-Wagner, Phys. Rev. Appl. 9, 064040 (2018).

[31] X. Z. Chen, R. Zarzuela, J. Zhang, C. Song, X. F. Zhou, G. Y. Shi, F. Li, H. A. Zhou, W. J. Jiang, F. Pan, and Y. Tserkovnyak, Phys. Rev. Lett. 120, 207204 (2018).

[32] T. Moriyama, W. Zhou, T. Seki, K. Takanashi, and T. Ono, Phys. Rev. Lett. 121, 167202 (2018).

[33] P. E. Roy, R. M. Otxoa, and J. Wunderlich, Phys. Rev. B 94, 014439 (2016).

[34] K. Olejník, T. Seifert, Z. Kašpar, V. Novák, P. Wadley, R. P. Campion, M. Baumgartner, P. Gambardella, P. Němec, J. Wunderlich, J. Sinova, P. Kužel, M. Müller, T. Kampfrath, and T. Jungwirth, Sci. Adv. 4, eaar3566 (2018). 
[35] N. Bhattacharjee, A. A. Sapozhnik, S. Y. Bodnar, V. Y. Grigorev, S. Y. Agustsson, J. Cao, D. Dominko, M. Obergfell, O. Gomonay, J. Sinova, M. Kläui, H.-J. Elmers, M. Jourdan, and J. Demsar, Phys. Rev. Lett. 120, 237201 (2018).

[36] J. Zhang, J. P. Velev, X. Dang, and E. Y. Tsymbal, Phys. Rev. B 94, 014435 (2016).

[37] J. M. Marmolejo-Tejada, K. Dolui, P. Lazić, P.-H. Chang, S. Smidstrup, D. Stradi, K. Stokbro, and B. K. Nikolić, Nano Lett. 17, 5626 (2017).

[38] Q. L. He, X. Kou, A. J. Grutter, G. Yin, L. Pan, X. Che, Y. Liu, T. Nie, B. Zhang, S. M. Disseler, B. J. Kirby, W. Ratcliff II, Q. Shao, K. Murata, X. Zhu, G. Yu, Y. Fan, M. Montazeri, X. Han, J. A. Borchers, and K. L. Wang, Nat. Mater. 16, 94 (2017).

[39] Q. L. He, G. Yin, L. Yu, A. J. Grutter, L. Pan, C.-z. Chen, X. Che, G. Yu, B. Zhang, Q. Shao, A. L. Stern, B. Casas, J. Xia, X. Han, B. J. Kirby, R. K. Lake, K. T. Law, and K. L. Wang, Phys. Rev. Lett. 121, 096802 (2018).

[40] S. Ghosh and A. Manchon, Phys. Rev. B 97, 134402 (2018).
[41] C.-X. Liu, X.-L. Qi, H. J. Zhang, X. Dai, Z. Fang, and S.-C. Zhang, Phys. Rev. B 82, 045122 (2010).

[42] R. S. K. Mong, A. M. Essin, and J. E. Moore, Phys. Rev. B 81, 245209 (2010).

[43] F. Freimuth, S. Blügel, and Y. Mokrousov, Phys. Rev. B 90, 174423 (2014)

[44] H. Li, H. Gao, L. P. Zârbo, K. Výborný, X. Wang, I. Garate, F. Doğan, A. Čejchan, J. Sinova, T. Jungwirth, and A. Manchon, Phys. Rev. B 91, 134402 (2015).

[45] S. Wimmer, K. Chadova, M. Seemann, D. Ködderitzsch, and H. Ebert, Phys. Rev. B 94, 054415 (2016).

[46] H. Kurebayashi, J. Sinova, D. Fang, A. C. Irvine, T. D. Skinner, J. Wunderlich, V. Novák, R. P. Campion, B. L. Gallagher, E. K. Vehstedt, L. P. Zârbo, K. Výborný, A. J. Ferguson, and T. Jungwirth, Nat. Nanotechnol. 9, 211 (2014).

[47] D. Culcer, A. MacDonald, and Q. Niu, Phys. Rev. B 68, 045327 (2003).

[48] S. Ghosh and A. Manchon, Phys. Rev. B 95, 035422 (2017). 